\title{
EXPRESIÓN SOCIO-AFECTIVA Y ARTE EN ESTUDIANTES DEL NIVEL PRIMARIO ${ }^{1}$
}

\section{Art and socio-affective expression of students in elementary school}

\author{
Patricia Liranzo Soto ${ }^{2} \bullet$ Rocío Hernández Mella ${ }^{3}$ \\ Andreína Jiménez Soto ${ }^{4} \bullet$ Berenice Pacheco Salazar ${ }^{5}$
}

Recibido: 30/4/2016 • Aprobado: 15/4/2017

\begin{abstract}
Resumen
El presente artículo surge de la experiencia de trabajo con 123 estudiantes del nivel primario, pertenecientes a cuatro centros educativos públicos en el municipio de Yamasá, República Dominicana. Se utilizó el instrumento Valoración del desarrollo socio-afectivo de Hernández \& Andújar (1999), para evaluar la dimensión socio-afectiva con relación a la expresión de sentimientos, en el cual la niña o el nińo debía reconocer la emoción implicada en una historia o situación relatada que ocurría en el entorno escolar. Se presentan aquí los resultados obtenidos tras la aplicación del citado instrumento, antes y después de la realización de una intervención denominada Encuentros Psico-Afectivos (EPA), consistente en la ejecución de talleres lúdicos y artísticos con el propósito de permitir la manifestación de la creatividad y generar la expresión de la afectividad de los niños y las niñas participantes. A través de un análisis comparativo de ambas mediaciones, se explica el cambio acaecido en la emocionalidad de los niños y las niñas participantes.
\end{abstract}

Palabras clave: arte; afectividad; educación primaria; desarrollo socio-afectivo.

1. Es la cuarta entrega.

2. Instituto Tecnológico de Santo Domingo (INTEC), República Dominicana.

Correo electrónico: patricia.liranzo@intec.edu.do

3. Instituto Tecnológico de Santo Domingo (INTEC), República Dominicana.

\begin{abstract}
This article comes from the experience of working with 123 students in the elementary level, from four public schools in the municipality of Yamasá, Dominican Republic. The instrument that was used was the Valoración del desarrollo socio-afectivo from Hernández \& Andújar (1999) to assess the socio-affective dimension in relation to the manifestation of feelings, in which the children had to recognize the emotion that was involved in a story or narrated situation that was given to them that occurs in the school environment. The results obtained by the administration of the previously mentioned instrument are presented here before and after performing an intervention called Encuentros Psico-Afectivos (EPA) or "Psycho-Affective Encounters (PAE)", which consisted in the execution of recreational and artistic workshops to allow the expression of creativity and to generate the manifestation of affectivity in the children who were participating. Through the comparative analysis of both interventions, there is a noticeable emotional change in the participating children.
\end{abstract}

Keywords: art; affectivity; elementary school; socio-affective development.
4. Instituto Tecnológico de Santo Domingo (INTEC), República Dominicana.

5. Instituto Tecnológico de Santo Domingo (INTEC), República Dominicana. 


\section{Introducción}

La dimensión afectivo-emocional es la base del desarrollo holístico del nińo y de la nińa, ya que implica la conexión entre lo interno, asumido como las condiciones fisiológicas, sensoriales y cognoscitivas, y lo externo, referido al comportamiento o conjunto de acciones (Hernández, 2012). Del mismo modo, el arte hace posible un espacio de búsqueda, reflexión y manifestación de las experiencias emocionales que horizontaliza la actividad individual y social, convirtiendo al ser humano en el artífice de sí mismo (Abad, 2009).

En ese sentido, el propósito de este trabajo consiste en describir la expresión afectiva de niños y niñas en su contexto escolar, antes y después de su participación en talleres lúdicos y artísticos, como uno de los componentes del estudio más amplio denominado Arte y afectividad: desarrollo de actitudes positivas y de una expresividad creativa en una experiencia escolar inclusiva. Este artículo es el cuarto correspondiente a una serie de cinco trabajos referidos a la investigación mencionada en líneas anteriores.

La afectividad se manifiesta por medio de las relaciones interpersonales desarrolladas en el espacio escolar. Piaget $(1986,1975)$ postula que todo acto humano incluye una energía afectiva que evoluciona simultáneamente con lo cognoscitivo, por lo cual ambos son manifestaciones esenciales de la integridad de la persona. De ahí que el ambiente escolar está llamado a propiciar el enriquecimiento de los vínculos afectivos por medio del reconocimiento, la designación y la expresión de los sentimientos (De Zubiria, 2007, citado por Villegas, 2010; Rodríguez, 2010).

El arte, por su parte, es la via regia de la expresión emocional, pues transforma la salud física y afectiva, generando valores y capacidades en las personas (Jiménez, 2010). Asimismo, el arte fomenta la creatividad, que representa una oportunidad para elevar la calidad de la educación (Villamán, 2003).
La investigación se realizó con un enfoque mixto, es decir, con un diseño que utilizó instrumentos cuantitativos y técnicas de recolección cualitativas. Se contó con un número de participantes de 123 niños y nińas de los grados de primero a cuarto del nivel básico, los cuales se seleccionaron a través de un muestreo no probabilístico de tipo intencional. Los participantes asistían a cuatro centros educativos del municipio de Yamasá de la regional educativa de Monte Plata, República Dominicana.

En esta oportunidad, se presentan los resultados obtenidos con la aplicación del instrumento Valoración del desarrollo socio-afectivo, que evalúa la dimensión socio-afectiva enfatizando en la expresión de los sentimientos, a partir de situaciones que ocurren en el contexto escolar. Cada situación del instrumento está diseñada para provocar una expresión afectiva que el niño o la niña fuese capaz de identificar. Para cada situación hay dos puntajes nominales: el total, que implica responder con el sentimiento esperado que la situación plantea, y el puntaje parcial, que supone señalar un sentimiento relacionado con la situación presentada. La categoría nula se refiere a la identificación de otro sentimiento que de ninguna manera responde a dicha situación.

\section{Resultados de la primera aplicación del instrumento Valoración del Desarrollo Socio- Afectivo}

Figura 1. Afectividad general de $1^{\circ}$ y $2^{\circ}$ grado (primera aplicación)

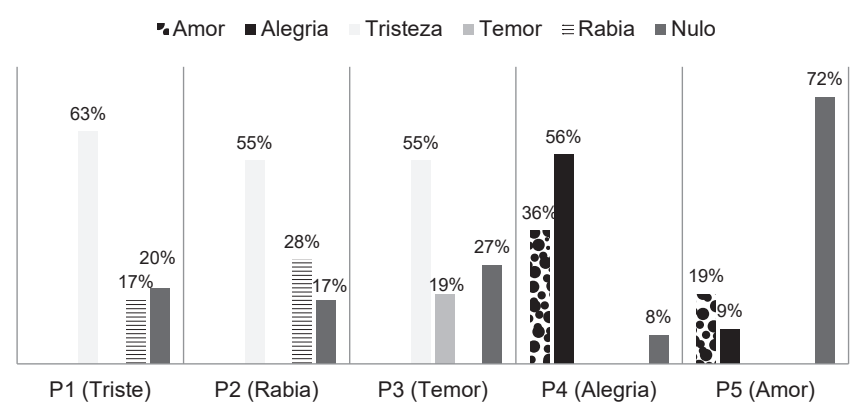


Los niños y las niñas de $1^{\circ}$ y $2^{\circ}$ grado identifican el sentimiento de tristeza como el de mayor manifestación, tanto en la situación donde este era el esperado (P1 63\%) como en aquellas situaciones donde era una respuesta de tipo parcial (P2 55\% y P3 55\%) y debían reconocer otras emociones como las preponderantes, que no fueron destacadas por los niños y las niñas, como son la rabia $(28 \%)$ y el temor (19\%).

En cuanto al sentimiento de alegría, el 56\% de los niños y las niñas logró identificarlo en la situación correspondiente (P4), no así el amor (P5) que solamente recibió el $19 \%$ de reconocimiento; mientras el $72 \%$ de los niños y las niñas de $1^{\circ}$ y $2^{\circ}$ grado de primaria fue de respuestas nulas.

Figura 2. Afectividad general de las nińas de $1^{\circ} y$ $2^{\circ}$ grado (primera aplicación)

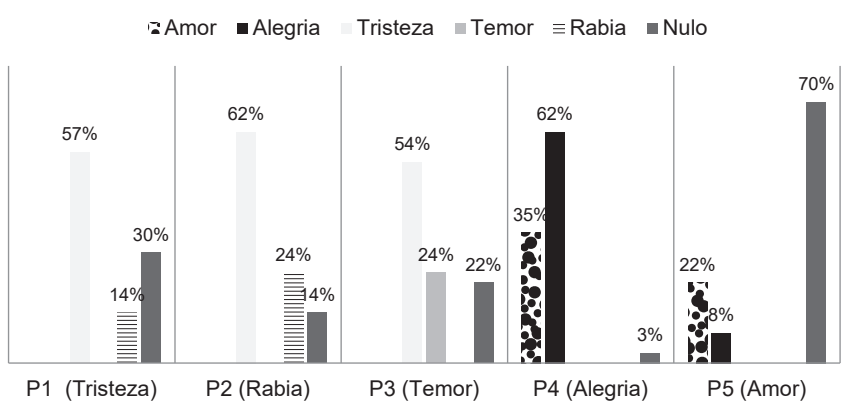

Las niñas de $1^{\circ}$ y $2^{\circ}$ grado de primaria presentan un comportamiento afectivo similar al descrito en la Figura 1, que corresponde a la totalidad de los y las participantes. Esto quiere decir que identifican la tristeza con un $57 \%$ en la P1, 62\% en la P2 y $54 \%$ en la $\mathrm{P} 3$, aunque en estas dos últimas no es el sentimiento esperado.

Con respecto a la situación en la P4, las niñas identifican en un $62 \%$ la alegría y en un $22 \%$ el amor en la P5, destacándose en un 70\% las respuestas nulas; es decir, la identificación de otros sentimientos que no corresponden ni al amor ni a la alegría.
Figura 3. Afectividad general de los nińos de $1^{\circ}$ y $2^{\circ}$ grado (primera aplicación)

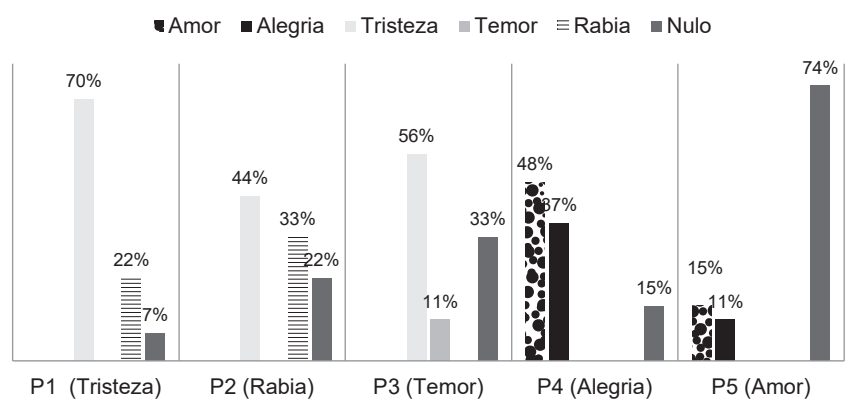

En los nińos de $1^{\circ}$ y $2^{\circ}$ grado de primaria se mantiene el patrón general del reconocimiento al sentimiento de tristeza, $70 \%$ para la P1, 44\% para la P2 y $56 \%$ para la $\mathrm{P} 3$, destacando que ellos manifiestan mayor tristeza que las niñas en la situación donde este sentimiento debe ser reconocido (P1).

Para las P4 y P5, referidas a la identificación de la alegría y el amor como sentimientos predominantes o totales, la frecuencia de reconocimiento es baja: $37 \%$ en la P4 y $15 \%$ en la P5, sobresaliendo, nueva vez, la respuesta nula en la $\mathrm{P} 5$ con un $74 \%$, ligeramente por encima de las niñas.

\section{Figura 4. Afectividad general de $3^{\circ}$ grado (pri- mera aplicación)}

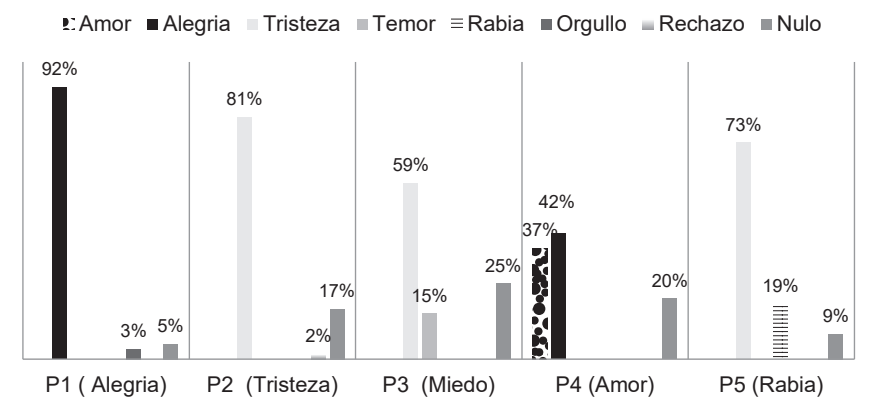

Los niños y las niñas de $3^{\circ}$ grado muestran una identificación correcta de los sentimientos para las P1 y P2, con $92 \%$ alegría y $81 \%$ tristeza, respectivamente. Para las P4 y P5, cuyos sentimientos a identificar eran temor y rabia, las respuestas fueron de tristeza en un $59 \%$ y $73 \%$, respectivamente. Para la P4 que indagaba acerca del sentimiento amor, 
este se presentó con un 37\%, mientras la alegría lo hizo con un $42 \%$.

Figura 5. Afectividad general de las niñas de $3^{\circ}$ grado (primera aplicación)

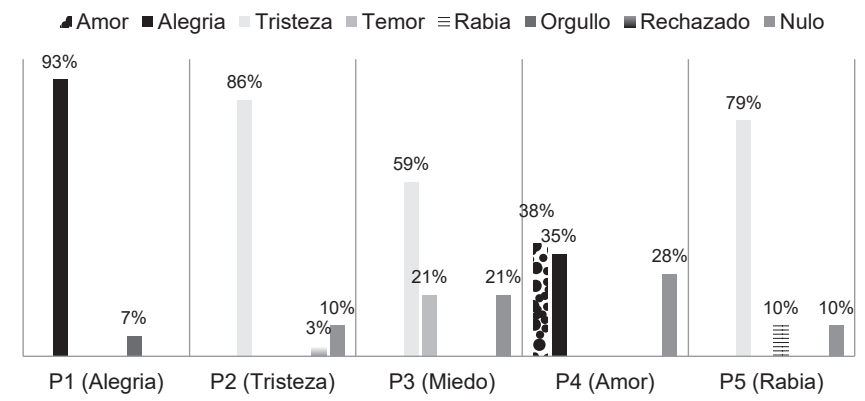

Las niñas de $3^{\circ}$ grado de primaria discriminan de manera correcta los sentimientos en las situaciones de las P1 y P2, correspondientes a la alegría con un $93 \%$ y a la tristeza con un $86 \%$, respectivamente, de manera congruente con los resultados generales. En cuanto a lo que se refiere a las P4 y P5, continúa repitiéndose el patrón general, esto es, una baja identificación de los sentimientos predominantes, consistentes en temor y rabia, mientras que la tristeza aparece con mayor frecuencia, 59\% y 79\%, respectivamente. Con relación a la $\mathrm{P} 4$, se muestra el amor con un 38\% como sentimiento dominante, y la alegría con un 35\%, como sentimiento parcial.

\section{Figura 6. Afectividad general de los niños de $3^{\circ}$ grado (primera aplicación)}

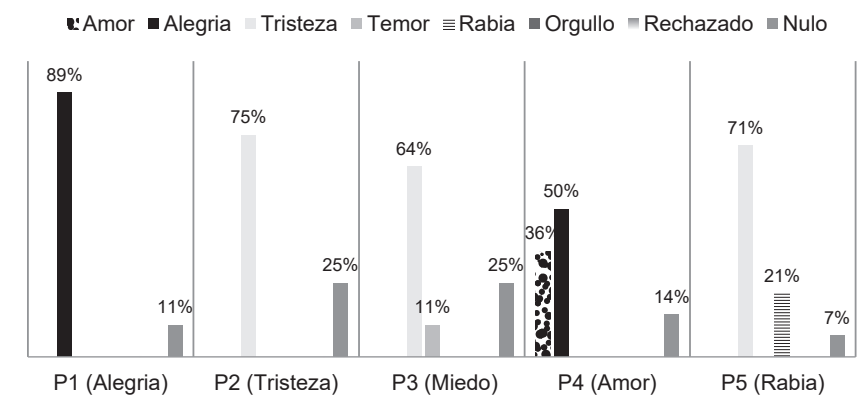

Los niños de $3^{\circ}$ grado de primaria son capaces de reconocer de manera adecuada los sentimientos en las situaciones de las P1 y P2, correspondientes a la alegría con un $89 \%$ y a la tristeza con un $75 \%$, respectivamente, en semejanza con los resultados generales, al igual que ocurrió con las niñas. En lo concerniente a las $\mathrm{P} 4$ y P5, continúa repitiéndose el patrón general, relativo a una baja identificación de los sentimientos predominantes, temor y rabia, en tanto que la tristeza aparece con mayor frecuencia, $64 \%$ y $71 \%$, respectivamente, tal y como ocurre con las niñas. Con referencia a la P4, se muestra el amor con un $36 \%$ y la alegría con un $50 \%$, es decir, un patrón invertido del sentimiento que debía predominar.

A partir de la finalización de la primera medición del aspecto socio-afectivo, junto con los demás instrumentos y las técnicas de recolección de datos cualitativos, se estableció la intervención bajo la modalidad de taller, la cual consistió en un conjunto de cuatro talleres alrededor del cuerpo que se denominó Encuentros Psico-Afectivos (EPA).

Estos talleres fueron creados con la concepción de que incorporar lo artístico y afectivo en la experiencia educativa propicia la expresión y el desarrollo de las potencialidades de todos los actores involucrados, convirtiendo dicha experiencia en motivadora, inclusiva, creativa y significativa.

El primer taller se titula El cuerpo vivencial; el segundo, El cuerpo que expresa; el tercero, El cuerpo que comunica; y el cuarto taller, El cuerpo que representa. En estos cuatro talleres se implementaron estrategias de intervención lúdicas y artísticas que complementaron y apoyaron el proceso enseñanza-aprendizaje de los niños y las niñas participantes. Las actividades que conformaron cada taller fueron una oportunidad que tuvieron niños y niñas para expresar su afectividad, tanto individual como grupal.

El propósito general de los talleres EPA fue desarrollar actitudes positivas y una expresividad creativa como herramientas para la construcción de una experiencia escolar inclusiva. Mientras que, de manera particular, se pretendía que los y las estudiantes pudieran reconocer y valorar su propio 
cuerpo como vía de expresión de la imaginación, las vivencias y las emociones.

\section{Resultados de la segunda aplicación del instrumento Valoración del Desarrollo Socio- Afectivo}

La investigación Arte y afectividad: desarrollo de actitudes positivas y de una expresividad creativa en una experiencia escolar inclusiva se programó para ser ejecutada a lo largo de diez meses. En el segundo mes, correspondiente a mayo de 2014, se efectuó la primera aplicación de instrumentos y técnicas de recolección de información, justo antes de la finalización del año escolar. Los talleres psico-afectivos se llevaron a cabo durante los meses de septiembre y octubre, una vez iniciado un nuevo período académico, mientras que la segunda aplicación del instrumento se ejecutó en el mes de noviembre de 2014, antes de las vacaciones de navidad.

De ahí que haya resultado que la primera aplicación del instrumento correspondiera a niños y niñas cursando los grados $1^{\circ}, 2^{\circ}$ y $3^{\circ}$, mientras que, en la segunda aplicación a los mismos estudiantes, ellos y ellas estaban cursando los grados $2^{\circ}, 3^{\circ}$ y $4^{\circ}$.

Figura 7. Comparación de la afectividad general de niñas y niños de $2^{\circ}$ y $3^{\circ}$ grado

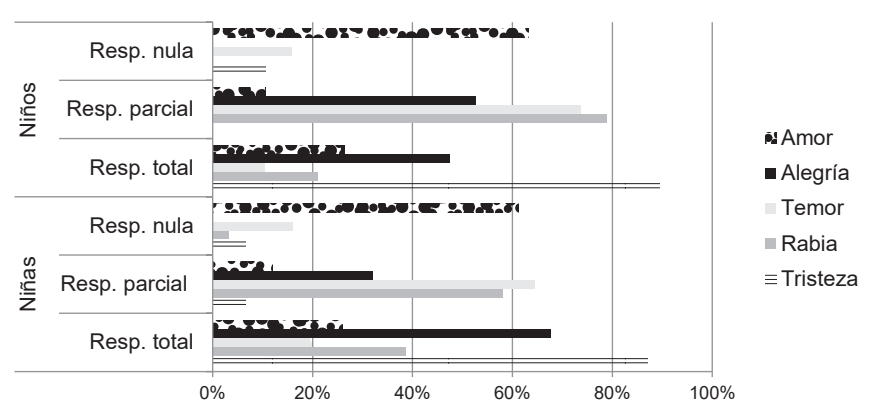

En la figura 7 resalta el sentimiento de tristeza como el más reconocido en la situación esperada, tanto en los niños, con un $90 \%$, como en las niñas, con un $87 \%$. El segundo sentimiento de mayor distinción es la alegría, con un 68\% para las niñas y un $48 \%$ para los nińos.

En las respuestas parciales, estas son, aquellas que indican un sentimiento que no es el esperado para la situación definida, pero sí es considerado como una posibilidad, la rabia y el temor son los más citados. En los niños la rabia obtuvo un 79\% y el temor un $74 \%$, mientras que en las nińas la relación se invierte, siendo el temor el sentimiento con mayor puntuación, $65 \%$, seguido de la rabia, con un $58 \%$.

La respuesta nula que más destaca es el amor, con un $63 \%$ en los niños y un $61 \%$ en las niñas.

\section{Figura 8. Comparación de la afectividad general de niñas y niños de $4^{\circ}$ grado}

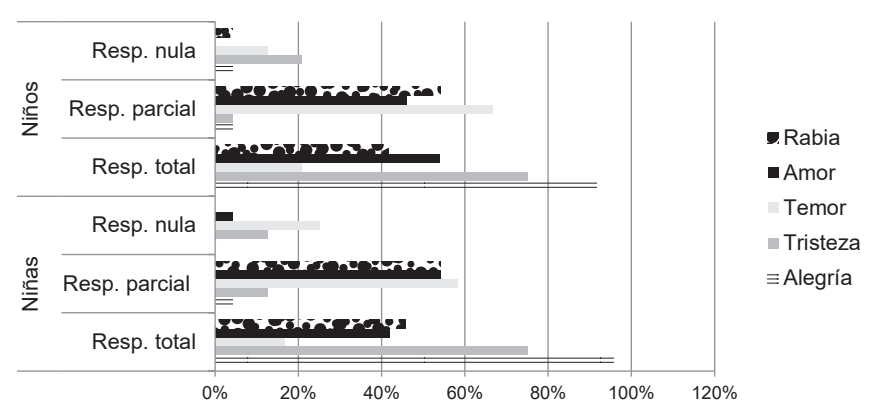

La figura 8 muestra a la alegría como el sentimiento de mayor reconocimiento, ya que en las nińas posee el $96 \%$ y en los niños un $92 \%$. Le sigue la tristeza que, en ambos, niños y niñas, se manifiesta con un $75 \%$. Llama la atención que el amor se presenta más alto en los varones, como respuesta total, en un $54 \%$ y en las niñas en un $42 \%$.

La respuesta parcial de mayor manifestación, tanto en los niños, con el 67\%, como en las niñas, con un $58 \%$, es el temor. A este sentimiento le sigue en importancia la rabia, con el mismo puntaje del $54 \%$ en los niños y las niñas. Para estas últimas, el amor se presenta con el mismo valor.

Conviene destacar que las respuestas nulas se presentan en puntajes menores al $25 \%$. 
Resultados comparativos entre la primera y la segunda aplicación del instrumento de Valoración del Desarrollo Socio-Afectivo

La relevancia de la comparación que se presenta a continuación radica en que da a conocer el efecto ejercido por los talleres en el reconocimiento de los sentimientos, por parte de los niños y las niñas.

Como se ha consignado, aparece una diferencia en los grados escolares entre la primera y la segunda aplicación, en vista de que la primera medida se llevó a cabo al final de un año escolar, mientras que la ejecución de los talleres y la segunda aplicación se efectuaron en el ańo siguiente y el instrumento debía utilizarse antes y después en los mismos niños y niñas.

Figura 9. Comparación de la afectividad general de niños y nińas de los primeros grados entre la primera y la segunda aplicación

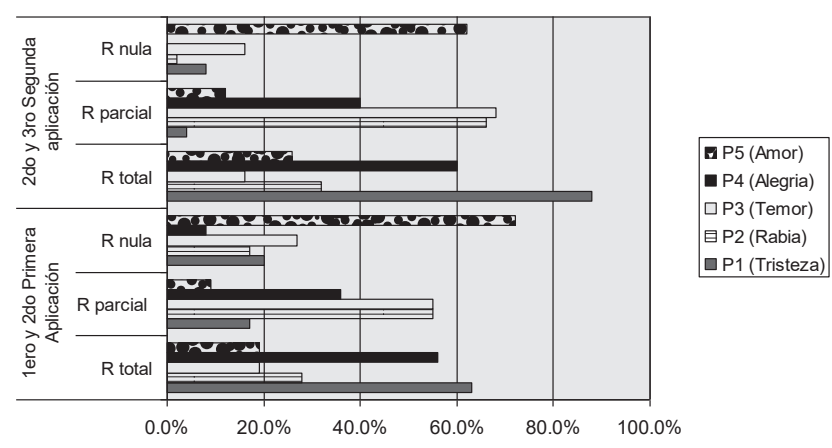

La figura 9 muestra un aumento de la respuesta total de reconocimiento, que es la pertinente en cada caso presentado en el instrumento, de los siguientes sentimientos: tristeza, de un $63 \%$ a un $88 \%$; rabia, del $28 \%$ al $32 \%$; alegría, del $56 \%$ al $60 \%$, y amor, del 19\% al 26\%; mientras que el temor mostró una disminución del 19\% al 16\%.

Con respecto a la respuesta parcial de reconocimiento de sentimientos, que no es la esperada, pero es aceptada, aumentaron las referidas a la rabia, del $55 \%$ al $66 \%$; el temor, del $55 \%$ al $68 \%$; la alegría, del $36 \%$ al $40 \%$, y el amor, del $9 \%$ al $12 \%$; mientras que la tristeza parcial disminuyó del $17 \%$ al $4 \%$.

En este punto, afirmamos que el temor suple su disminución en la respuesta total con su aumento en la parcial, mientras con la tristeza ocurre lo inverso; es decir, que ambos sentimientos compensan en la segunda aplicación.

Todas las respuestas nulas disminuyeron en la segunda aplicación, efectuada luego de los talleres psico-afectivos, lo que comprueba su efectividad en el reconocimiento de los sentimientos, por parte de los niños y las niñas participantes.

Figura 10. Comparación de la afectividad general de niños y nińas de grados posteriores entre la primera y la segunda aplicación

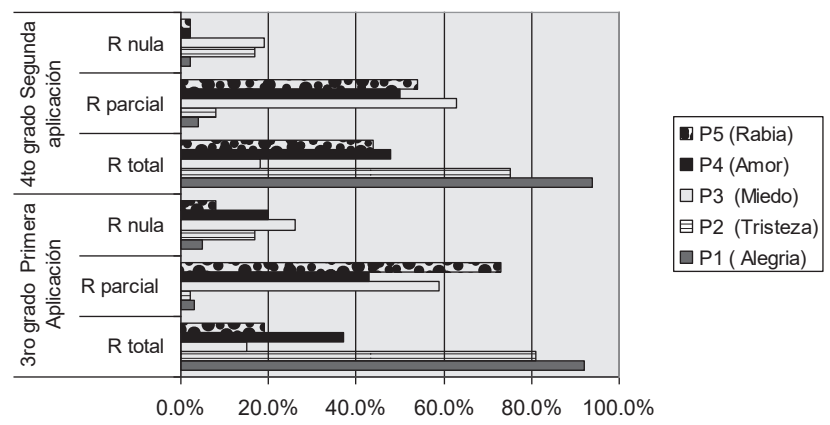

La figura 10 muestra un aumento en las respuestas totales, producido en la segunda aplicación del instrumento socio-afectivo, consignado a los siguientes sentimientos: alegría, del $92 \%$ al $94 \%$; miedo, del $15 \%$ al $18 \%$; amor, del $37 \%$ al $48 \%$, y rabia, del $19 \%$ al $44 \%$, mientras que la tristeza disminuyó del $81 \%$ al $75 \%$ en su respuesta total.

En lo relativo a las respuestas parciales, la segunda medición mostró aumentos en los siguientes sentimientos: miedo, de 59\% a 63\%; amor, de $43 \%$ a $50 \%$; alegría, de $3 \%$ a $4 \%$, y tristeza, de $2 \%$ a $8 \%$. El sentimiento de rabia disminuyó de $73 \%$ a $54 \%$. 
Tabla 1. Variación en el efecto de los sentimientos entre la primera y la segunda aplicación del instrumento en nińos y niñas de los primeros grados

\begin{tabular}{lccccc}
\hline $\begin{array}{l}\text { Niños y niñas } 1^{\circ} \text { y } 2^{\circ} \text { grado } \\
\text { Primera medición }\end{array}$ & \multicolumn{5}{c}{$\begin{array}{l}\text { Niños y niñas } 1^{\circ} \text { y } 2^{\circ} \text { grado } \\
\text { Segunda medición }\end{array}$} \\
\hline Sentimientos & M & DT & M & DT & $d$ Cohen \\
\hline Respuesta total & 0.37 & 0.21 & 0.44 & 0.29 & -0.276 \\
Respuesta parcial & 0.34 & 0.21 & 0.38 & 0.30 & -0.154 \\
\hline
\end{tabular}

La tabla 1 muestra un aumento en la respuesta total de $\mathrm{M}=0.37$ (Media) DS=021(Desviación standard) a $M=0.44 \mathrm{DS}=0.29$. En la respuesta parcial, los valores se incrementaron de $\mathrm{M}=0.34 \mathrm{DS}=0.21 \mathrm{a}$ $\mathrm{M}=0.38 \mathrm{DS}=0.30$. Ambos tipos de respuestas con una $\mathrm{d}$ Cohen $=-0.276$ y d $=-0.154$, respectivamente, siendo en la respuesta total donde se evidencia un tamaño del efecto con una pequeña magnitud.

Tabla 2. Variación en el efecto de los sentimientos entre la primera y la segunda aplicación del instrumento en niños y niñas de los grados superiores

\begin{tabular}{lccccr}
\hline $\begin{array}{l}\text { Niños y niñas } 3^{\circ} \text { grado } \\
\text { Primera medición }\end{array}$ & \multicolumn{5}{c}{$\begin{array}{l}\text { Niños y niñas 4o grado } \\
\text { Segunda medición }\end{array}$} \\
\hline Sentimientos & M & DT & M & DT & $d$ Cohen \\
\hline Respuesta total & 0.49 & 0.35 & 0.56 & 0.29 & -0.217 \\
Respuesta parcial & 0.36 & 0.32 & 0.36 & 0.27 & 0 \\
\hline
\end{tabular}

La tabla 2 exhibe un aumento en la respuesta total de $\mathrm{M}=0.49 \mathrm{DS}=0.35$ a $\mathrm{M}=0.56 \mathrm{DS}=0.29$, con un $\mathrm{d}$ Cohen $=-0.217$, correspondiente a un tamaño del efecto de pequeña magnitud. En la respuesta parcial no hubo incremento en los valores.

Tabla 3. Variación en el efecto de los sentimientos de alegría y amor entre la primera y la segunda aplicación del instrumento en niños y niñas de los primeros grados

\begin{tabular}{lccccc}
\hline $\begin{array}{l}\text { Niños y niñas } 1^{\circ} \text { y } 2^{\circ} \text { grado } \\
\text { Primera medición }\end{array}$ & \multicolumn{5}{c}{$\begin{array}{l}\text { Niños y niñas } 2^{\circ} \text { y } 3^{\circ} \text { grado } \\
\text { Segunda medición }\end{array}$} \\
\hline Sentimientos & M & DT & M & DT & $d$ Cohen \\
\hline Alegría & 0.49 & 0.14 & 0.50 & 0.14 & -0.285 \\
Amor & 0.14 & 0.07 & 0.19 & 0.09 & -0.620 \\
\hline
\end{tabular}

La tabla 3 especifica el tamaño del efecto para los sentimientos alegría y amor. El primero con una $\mathrm{M}=0.46 \mathrm{DS}=0.14$ aumentó a $\mathrm{M}=0.50 \mathrm{DS}=0.14$. El segundo con una $\mathrm{M}=0.14 \mathrm{DS}=0.07$ incrementó a $\mathrm{M}=0.19 \mathrm{DS}=0.09$. El tamańo del efecto en la alegría fue pequeño, con un $\mathrm{d}=-0.285$, mientras que el amor presentó una $\mathrm{d}=-0.620$, correspondiente a un tamaño del efecto moderado.

Tabla 4. Variación en el efecto de los sentimientos de amor entre la primera y la segunda aplicación del instrumento en niños y nińas de los grados superiores

\begin{tabular}{lccccc}
\hline $\begin{array}{l}\text { Niños y niñas } 3^{\circ} \text { grado } \\
\text { Primera medición }\end{array}$ & \multicolumn{5}{c}{$\begin{array}{l}\text { Niños y niñas } 4^{\circ} \text { grado } \\
\text { Segunda medición }\end{array}$} \\
\hline Sentimientos & M & DT & M & DT & $d$ Cohen \\
\hline Amor & 0.40 & 0.04 & 0.49 & 0.01 & -3.08 \\
\hline
\end{tabular}

La tabla 4 indica la variación del efecto para el sentimiento amor, con una $\mathrm{M}=0.40 \mathrm{DS}=0.040$ que aumentó a $\mathrm{M}=0.49 \mathrm{DS}=0.01$. La variación del efecto en este sentimiento fue muy grande, con una $\mathrm{d}=-3.08$.

\section{Discusión}

El reconocimiento de distintos sentimientos se evaluó a partir de la presentación de una historia en la cual el niño y la niña debían señalar la emoción involucrada. En esta investigación, se llevó a cabo una medición inicial antes de la intervención efectuada con los talleres Encuentros Psico-Afectivos (EPA) y una segunda evaluación luego de dichos eventos, con el fin de valorar los cambios ocurridos en los niños y las niñas participantes, con respecto a su capacidad para identificar sentimientos.

En el caso de los grados inferiores, los sentimientos a identificar fueron: amor, alegría, tristeza, temor y rabia; para los grados superiores, los sentimientos a señalar fueron: amor, alegría, tristeza, temor, rabia, orgullo y rechazo. 
En la evaluación inicial, para los niños y las niñas de los grados inferiores de las cuatro escuelas abordadas, la capacidad de reconocer la emoción esperada de tristeza fue importante, seguida por la alegría; sin embargo, la identificación de otras emociones esperadas resultó leve, ya que la tristeza era la respuesta adjudicada a situaciones en las que no correspondía hacerlo.

En la segunda medición de la socio-afectividad para los grados inferiores se profundiza la aparición del sentimiento de tristeza, tanto en los casos que se esperaba como respuesta, como en aquellos donde se admitía como una contestación parcialmente aceptable.

Es preciso hacer un alto y reflexionar sobre la preeminencia del sentimiento de tristeza en estos estudiantes que apenas inician su escolaridad, ya que estos hallazgos evidencian cómo se sienten los niños y las niñas con ellos mismos y frente a la escuela.

Cabe destacar que, frente al reconocimiento del sentimiento de amor, los nińos y las nińas de los grados inferiores del nivel básico presentaron una respuesta nula muy elevada en la primera evaluación, lo que podría significar una aparente dificultad para identificar esta emoción. Para el caso de la segunda estimación en estos mismos niños, la identificación del amor aumentó, pero solo ligeramente.

En cuanto a la afectividad en el grado superior, en la evaluación inicial los niños y las niñas fueron capaces de reconocer los sentimientos de alegría y tristeza esperados, no así los demás: amor, temor y rabia.

$\mathrm{Al}$ igual que en los grados inferiores, la tristeza como respuesta atribuida es elevada, lo que confirma que estos niños y estas niñas de los grados superiores también se sienten tristes en su interior y así lo proyectan.
Si bien es verdad que el amor tuvo una manifestación leve, es decir, fue identificado con más precisión que en los grados anteriores, las respuestas nulas también fueron considerables. $\mathrm{Al}$ analizar la segunda medición, aumenta el reconocimiento de la alegría, aunque baja ligeramente la atribución apropiada al sentimiento de tristeza. Lo más destacable es la manera en que el sentimiento de rabia logra prácticamente triplicarse en las respuestas adecuadas de reconocimiento.

Rodríguez (2010) y Villegas (2010) nos arrojan alguna luz para comprender este comportamiento, ambos plantean que a medida que los seres humanos crecen el control de las emociones se torna más interno que externo. Sin embargo, es De Zubiria (2007, citado por Villegas, 2010) quien releva que, a pesar de esto, existen dificultades en el manejo emocional debido a la complejidad que al crecer y desarrollarse el individuo encuentra en su relación emocional, al estar implicado en el contacto e intercambio con las otras personas. Quizás es por esa dificultad que la identificación de varios sentimientos no ocurrió o disminuyó.

De Zubria (2007) también propone que la forma para desarrollar las relaciones debe pasar por tres acciones: conocer, valorar e interactuar; todas pueden ser ejecutadas en el contexto educativo y se ejercitaron con los talleres EPA efectuados para los estudiantes que participaron en esta investigación.

En la primera aplicación, los niños de los grados inferiores fueron capaces de reconocer el sentimiento de tristeza que se encuentra en las situaciones expuestas, pero las niñas proyectan más su tristeza en situaciones asociadas al reconocimiento de otros sentimientos.

Para la segunda medición, la tristeza en las niñas aumentó hasta prácticamente equiparar la de los niños, a la vez que continuaban atribuyendo la tristeza a las situaciones donde debían reconocer otros sentimientos. ¿Qué significa esto? Se advierte que los niños y las niñas viven con la tristeza y que estas 
últimas son más tristes que los primero. También vale decir que, a estas niñas se les dificulta sentir, debido a la opresión que el contexto violento en el que viven ejerce sobre ellas, y esa indefensión se transforma en tristeza, como una manera incipientemente de defensa.

Adicionalmente, en la segunda estimación se halla en estas niñas un aumento significativo del sentimiento de rabia, mientras que en los niños esta emoción disminuyó. La rabia es, de hecho, un sentimiento más complejo que la alegría o la tristeza y, en este caso, las niñas muestran un logro mayor en el desarrollo socio-afectivo que los niños, luego de haber participado en los talleres EPA.

$\mathrm{Al}$ evaluar la medida inicial obtenida en el grado superior, las nińas superan levemente a los varones en la alegría y moderadamente en el reconocimiento del sentimiento de tristeza, contrario a lo encontrado en los grados inferiores. Esta paradoja puede explicarse considerando que la alegría reconocida es en realidad una defensa adaptativa de ese contexto opresor, que comienza a formarse en los grados inferiores y se consolida en los siguientes grados como una especie de alegría superficial.

En la segunda medición, tanto niños como niñas elevaron su reconocimiento de la alegría a valores casi máximos. La rabia aumenta significativamente en ambos géneros, pero el valor en las niñas se amplía de manera descomunal. Un cambio importante ocurrió en el reconocimiento del amor por parte de los varones, que aumentó mucho más que en las niñas.

Estos hallazgos podrían ser considerados como un indicio del valor de los talleres EPA para promover el desarrollo socio-afectivo. Aun si la alegría se utilizara como escudo de defensa contra la violencia es una mejor respuesta a la agresión, es decir, recurrir a la alegría como una alternativa de comunicación es mucho más sano. La rabia se va reconociendo y, como suele suceder, las niñas se adelantan a los varones en madurez emocional. Por último, resulta conmovedor el que sean los varones quienes muestren ser más sensibles al reconocimiento del sentimiento del amor. En este sentido, surge una posible explicación que indica que la atención que recibieron por parte de las y los facilitadores de los talleres EPA, así como el efecto ejercido por las dinámicas desarrolladas, hayan podido contribuir con este cambio.

Los talleres EPA, como intervención lúdica y artística, han generado un efecto que, aunque resultara reducido, favorece la capacidad de identificar todos los sentimientos correspondientes a las situaciones esperadas. Para los niños y las niñas de grados inferiores los talleres tuvieron un impacto leve en el señalamiento del sentimiento de alegría y uno moderado en el reconocimiento del sentimiento de amor. Los niños y las niñas de grados superiores mostraron un efecto mayor, aunque de magnitud pequeña, en la verificación del sentimiento de amor.

Estos hallazgos apoyan la propuesta de Rodríguez (2010) y de Villegas (2010) en el sentido de que una manera apropiada e imprescindible de lidiar con las emociones es reconocerlas y nombrarlas. Esto posibilita el que se pueda pensar y conversar sobre ellas, y compartirlas con las figuras importantes; para el logro de estos fines contribuyeron la investigación y la intervención desarrolladas.

\section{Recomendación}

Al verificar que los talleres Encuentros PsicoAfectivos (EPA) han tenido un efecto positivo hacia una mayor capacidad de reconocimiento de las emociones y los sentimientos por parte de los nińos y las niñas participantes en este estudio, hace que sea obligatorio recomendar su aplicación de manera más continua e integrada a la estructura escolar, para que los beneficios logrados puedan ampliarse a todos los actores de la experiencia educativa. 
En un próximo artículo, las autoras estarían divulgando con cierto grado de detalle informaciones sobre cada uno de los cuatro talleres EPA con los cuales se produjo la intervención y las actuaciones infantiles durante los mismos, aunque se adelanta la noticia de que la investigación tuvo como uno de sus productos la publicación de una guía dirigida a los docentes que recoge la manera de aplicar cada taller.

Debido a que los talleres EPA son dinámicas con sentido lúdico y artístico, el efecto que se ha podido lograr con los mismos remite a Pozzoli (2007), quien argumenta que el arte, en sus diversas manifestaciones, es una expresión de la interioridad de las personas y posee la capacidad de reconocer y reconstruir el ser profundo.

\section{Referencias}

Abad, J. (2009). Usos y funciones de las artes en la educación y el desarrollo humano. En Jiménez, L., Aguirre, I. \& Pimentel, L. (Coords.) Educación Artística Cultura y Ciudadania, 1-183. España: OEI. Recuperado de http://www.oei.es/ metas2021/EDART2.pdf

Borthwick, A. (2011) Children's perceptions of, and attitudes towards, their mathematics lessons. C. Smith. (Ed.), Proceedings of the British Society for Research into Learning Mathematics, 31(1), 37-43. Recuperado de http://www.bsrlm.org.uk/IPs/ip311/BSRLM-IP-31-1-07.pdf

Gardner, H. (1997). La mente no escolarizada: Cómo piensan los niños y cómo deberían enseñar las escuelas. México-España: Biblioteca del Normalista.

Hernández Mella, R. (2014). Aportes de la Psicología Social al análisis de la condición de sobreedad en la educación: De la exclusión a la oportunidad. (Tesis doctoral, inédita). Universidad Complutense de Madrid, España.
Hernández Mella, R. \& Pacheco, B. (2012). Vivir feliz como propuesta para un quehacer docente imaginativo e inclusivo. Santo Domingo: OEI y MINERD.

Hernández Mella, R. \& Andujar, C.; Scheker, A. \& Pérez M., \& Amiama, C. (2000). Instrumento de evaluación psicopedagógica. Santo Domingo: Secretaría de Estado de Educación.

Hernández Mella, R. \& Andújar, C. (1999). Valoración del desarrollo socioafectivo: instrumento, resultados y conclusiones. Santo Domingo. Secretaría de Estado de Educación.

Hernández Mella, R. (2012). Ambientes afectivos y efectivos de aprendizaje. Foro de Innovación en la Docencia. Xalapa, Veracruz: Facultad de Pedagogía Xalapa. Universidad Veracruzana. Recuperado de http://www.uv.mx/personal/rubhernandez/files/2012/02/Amb_afectivos_efectivos.pdf

Jiménez, L. (2010). Arte, cultura y ciudadanía: hacia la construcción de valores. B. Toro \& A. Tallone. (Eds.). Educación, valores y ciudadanía. Metas Educativas 2021. Madrid: OEI \& Fundación SM.

Piaget, J. (1975,1986). Seis estudios de Psicología. Barcelona: Barral Editores.

Restrepo, G. (2009).Contextosafectivosycognitivos en los procesos de aprendizaje. Revista Complutense de Educación, 20(1), 195-204. Recuperado del sitio web: http://revistas.ucm.es/index.php/RCED/article/ view/RCED0909120195A/15425

Rodríguez, G. (2010). Sentimientos y actitudes en la escuela. Capítulo 15, Educación, valores y ciudadanía, Organización de Estados Iberoamericanos para la Educación, la Ciencia y la Cultura. Recuperado de http://www.oei.es/metas2021/valoressm.pdf

Sánchez, S. \& Mesa, C. (1998). Actitudes hacia la tolerancia y la cooperación en ambientes multiculturales: evaluación e intervención educativa en un contexto concreto: la ciudad de Melilla. 
Series Monográficas Eirene, Vol 9., 1-36. España: Universidad de Granada. Recuperado de http:// www.ugr.es/ - eirene/publicaciones/item9/eirene9cap1.pdf

Villamán, M. (2003). Reinventar la escuela: algunas posibles provocaciones. En Centro Poveda (Eds.). Reinventar la escuela: ¿Qué opciones? Reflexiones sobre el futuro de la educación dominicana, (pp. 67-106). Recuperado de http://www.minerd.gob.do/idec/ Docs5/Reinventar\%20la\%20escuela.pdf

Villanueva, E. (2013). Danza y educación. En Ministerio de Educación de República Dominicana (Eds.). Propuestas ante los cambios que requiere la actualización curricular: naturaleza, contenidos y competencias. VIII Foro Nacional Educación Artística, Santo Domingo.

Villegas, C. (2010). La afectividad como el eje central del encuentro educativo. Ponencia presentada en Congreso Iberoamericano de Educación Metas Educativas 2021. Organización de los Estados Iberoamericanos para la Educación, la Ciencia y la Cultura. Recuperado de http://www.chubut.edu.ar/descargas/secundaria/ congreso/METAS2021/R0734b_Poster_Villegas.pdf

\section{Datos de filiación}

Patricia Liranzo Soto. Es psicóloga clínica, profesora e investigadora del Área de Ciencias Sociales y Humanidades del Instituto Tecnológico de Santo Domingo (INTEC). Posee una maestría en el área de Psicología Clínica por la Universidad Autónoma de Santo Domingo; licenciatura en Psicología; curso Experto en Peritaje Psicológico e Intervención Judicial; curso en Diagnóstico y Rehabilitación Neuropsicológica en Neurología Conductual; curso en Evaluación e Intervención Psicofisiológica en Medicina Conductual. Entre sus publicaciones están: Las diosas sometidas: autoconcepto en mujeres de grupos vulnerables. Encuentros afectivo-participativos y sus efectos en el self (2013); Apego y autoestima estudiantes femeninas en Psicología (2012); El proceso de descentralización escolar y sus condiciones de posibilidad (2006). Correo electrónico: patricia.liranzo@ intec.edu.do

Rocío Hernández Mella. Profesora-investigadora del Área de Ciencias Sociales y Humanidades del Instituto Tecnológico de Santo Domingo, INTEC. Doctora en Psicología Social; maestría en Planificación y Administración de la Educación; especialización en Psicología Educativa y licenciatura en Psicología. Entre sus publicaciones recientes están: Las diosas sometidas: Autoconcepto en mujeres de grupos vulnerables. Encuentros afectivo-participativos y sus efectos en el self(junto a Patricia Liranzo, 2014), Vivir feliz como propuesta para un quehacer docente imaginativo e inclusivo (junto a Berenice Pacheco, 2012); El arte salva (capítulo, 2012). Líneas de investigación: Psicología; calidad y procesos educativos. Correo electrónico: rocio.hernandez@intec. edu.do

Aurora Andreína Jiménez Soto. Es egresada de la carrera de Psicología de Instituto Tecnológico de Santo Domingo (INTEC), maestría en Gerencia Educativa en UNIBE; especialidad en Educación Artística, Cultura y Ciudadanía. Actualmente se desempeña como Técnico Docente Nacional del Área de Educación Artística de la Dirección General de Currículo del Ministerio de Educación; profesora de INTEC. Correo electrónico: aajsoto@gmail.com

BerenicePacheco-Salazar. Es doctora en Educación por la Universidad de Sevilla. Es licenciada en psicología con maestría en Género, Investigación y Desarrollo. Docente de la Universidad INTEC. Líneas de investigación: la violencia escolar; género $\mathrm{y}$ violencia, atención a la diversidad, y convivencia y mejora de las instituciones educativas. Correo electrónico: bereniceps@gmail.com 